\title{
I3- A New Intelligent Generation for Internet Networks
}

Nadia Boukhatem ${ }^{1}$, Benoit Campedel ${ }^{2}$, Hakima Chaouchi ${ }^{3}$, Vincent Guyot ${ }^{1,3}$, Francine Krief ${ }^{4}$, Thi Mai Trang Nguyen ${ }^{1,3}$, and Guy Pujolle ${ }^{3}$ 1 ENST, 2 ISEP, 3 LIP6, 4 LIPN

\begin{abstract}
This paper introduces new concepts to be applied for future Internet generations to better manage, react and control the network. These concepts are based on the intelligence aspect and the policy-based networking management paradigm. We first describe a policy-based networking management environment and some new protocols allowing an easy control of the QoS and the mobility. Then, we describe the Internet Third Generation (I3) platform that is our suggestion for an intelligent agent-based implementation of the policy based networking management.
\end{abstract}

Keywords: Network Policy Management, Policy server, PDP, PEP, COPS, SLS, SLA, COPS-SLS, Mobile IP, Multi-Agent system, AALAADIN, MADkit Platform.

\section{INTRODUCTION}

During the $80 \mathrm{~s}$, most academic centers exchanged messages via the "Net". The period 1990 through 2000 saw the arrival, for the public at large, of commercial offers allowing communications with high-speed servers. This was the Internet called Internet First Generation (I1). The Internet I1 is the system that we are using nowadays from our terminals. The Internet called 12, Internet Second Generation arrived at the beginning of the year 2000. A new Internet, called Third Generation (I3) of which primary concepts are described in this paper.

I1 comes from the initial work achieved by some scientists in the world believing in a universal communicating system. However, in that Internet throughputs were too slow, servers do not offer any guarantee to deliver the desired information. At the end of the $90 \mathrm{~s}$, the promises of increase of the

The original version of this chapter was revised: The copyright line was incorrect. This has been corrected. The Erratum to this chapter is available at DOI: 10.1007/978-0-387-35584-9_19 
throughput are real; costs become almost accessible and many new and attractive services appeared.

I2 arrives with its high throughput, its procession of services, its best stability against breakdowns and its ubiquitous access.

Between 2005 and 2010, the passage to I3 intends to lead to a more stable system and protocols, imperceptible breakdowns to the user, a large number of available multimedia applications, and a generalized access.

What directions to push on? The intelligence. The computer intelligence debate is not new. We believe that the system must be intelligent because the distributed nature of the network management requires an additional component: the time; the system must react on real time to unexpected situations and has to make decisions and adapt itself to a new situation and to control critical states automatically.

Intelligence is the chosen word to group several actions well distinct: to learn, communicate and infer. Several ways can be envisaged, with their own roots: Intelligent Networks (IN), smart networks and active networks. Intelligent Networks form an already old architectural concept where the intelligent word meant adaptation. The system has to be adaptable to the user demand. Even if some experiences were tested in telecommunication systems, we are still only in the first steps. In this area, the data-processing could induce a too large delay when using languages like Java that disseminate pieces of software in a very large number of points. These pieces of software can possibly move by themselves.

The second field is supported by smart networks. It is necessary to develop specific components to bring their expertise to solve problems and to control infrastructures and flows. As a first answer, there are some known solutions: they come from the intelligent agents field. An agent is an autonomous entity able to communicate with other agents, to perceive and to represent its environment. The whole agents in interaction form a multiagent system. Many criteria allow to classify these systems: the size of agents, the number of agents in interaction, the mechanisms and the types of communication, the behavior, the organization and the representation of the environment.

The third area corresponds to active networks. The intelligence in active networks, situated inside the nodes of the system, as well as in the user workstations, can adapt the nodes and the workstation to the type of information received. For example, the header of the packets can contain programs that must be executed in the routers. The nodes have to fit instantaneously to the arriving packet, what seems possible with new reconfigurable processors.

I3 research project focus on an intelligent-based control scheme belonging to the second class mentioned above (smart networks). The basic 
problem concerns the architecture of the system to integrate the "intelligent" components, so that they could be simple and cooperating to achieve the policy-based network management.

This paper presents the development of an intelligent platform based on a policy-based networking management system. Section 2 introduces policy concepts. Section 3 deals with a new protocol for controlling the configuration of hosts. Section 4 introduces the policy based mobility management within the global system. Then, section 5 is introducing the $\mathrm{I} 3$ model and platform

\section{AN INTRODUCTION TO POLICIES}

Policies can be defined as a "set of rules to manage and control access to network resources". The works dealing with policies come from different domains, sharing common needs. The most advanced works are found in the quality of service (QoS) domain, with the definition of a new protocol (COPS) and a new architecture. To make this specific approach more general, a working group has been created by the IETF to specify the information model to be used, as well as the general architecture. The goal of the information model is to define a general model that can be specialized into different domains, independently of any equipment or implementation.

The Policy Core Information Model (PCIM) is an extension to the DMTF CIM model [ref]. The network is seen as a state-machine, policies being used to control state changes. It must be able to identify/model the current state (objects) and define possible transitions (rules). This model simply defines rules as a set of conditions and a set of actions. An action is being activated when a rule condition is verified. The model includes a few more elements, to be able to define roles, priorities and execution order, but stays in a quite abstract form concerning objects.

Current works around QoS define two levels of extension: the QoS Policy Information Model (QPIM) and the QoS Device Datapath Information Model (QDDIM). The first one integrates QoS specific notions, to be able to create formal representations of abstract policies, such as: "If packet's protocol is http and its destination is in the EXECUTIVES user group, then assign IPP 7 to the packet header". To this aim, the model defines Policy Actions (RSVP - Resource Reservation Protocol, provisioning, PHB - Per

Hop Behavior) and traffic profiles, to specify processing to be done on requests and flows. The QDDIM model extends the previous one to define actions to be done on the equipments (configuration), where QPIM defines actions to be done on packets. 
The architecture defines a centralized model for management, policies storage, decision-making, and configuration distribution. This architecture is described in Figure 1.

The Policy Decision Point (PDP) is responsible for making decisions, to its own initiative, or by reacting to a request from a network element. It has to determine which configurations need to be applied to the resources to satisfy the network policies. The main functions deals with the determination of what rules are relevant to the different Policy Enforcement Points (PEPs), the conversion in an adapted format (PIB - Policy Information Base, MIB Management Information Base, etc.) and the guarantee of their right distribution.

A PEP is a logical entity that enforces policy decisions. It corresponds to a resource offering services that can be used to apply network policies. Main functions of a PEP consist to link the external representations (PIB, MIB, etc.) to the internal equipment configurations, and to maintain local policies integrity while monitoring their enforcement. When integrated in an admission control entity, the PEP is also responsible for validating the requests (ex. RSVP) by soliciting the PDP.

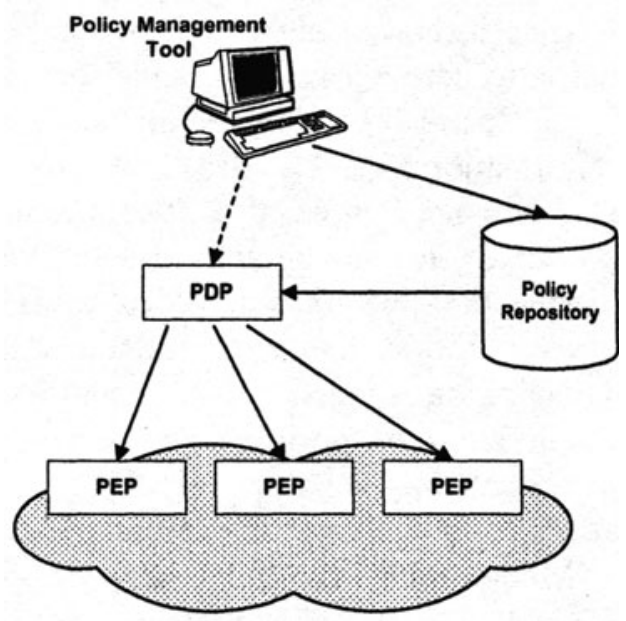

Figure 1. Policy-Based Management Architecture

The architecture model does not require any specific communication protocol or storage method for policies. Nevertheless, the COPS protocol and directories using Lightweight Directory Access Protocol version 3 (LDAPv3) seem to be the preferred ways. 


\section{COPS AND COPS-SLS}

In this section, we present a new protocol we developed to dynamically control SLS negotiation between the network provider and its customers. This protocol named as COPS-SLS is an extension of COPS protocol. It gives $\mathrm{I} 3$ platform a means to negotiate (with the network provider) the client's needs, such as QoS parameters, security requirements, mobility needs, etc..

\subsection{COPS protocol}

COPS (Common Open Policy Service) [1] is a simple request/response protocol based on TCP, proposed by the IETF Resource Allocation Protocol working group to convey policy information between the PEP and the PDP.

The PEP is the policy client and the PDP is the policy server. The local PDP is an optional entity acting as a cache. When the PEP needs policies to manage network resources, it sends a Request (REQ) message to the PDP. Upon receipt of the Decision (DEC) message from the PDP, it enforces network policies.

Two models are defined in policy control: Outsourcing model and Configuration model, a.k.a. Provisioning (PR) model [3]. In Outsourcing model (e.g. COPS-RSVP), the PEP does not know policies before the arrival of a resource request event. When a resource request arrives (e.g. a RSVP message arrives at an RSVP router), the PEP sends a REQ message to the PDP to obtain the decision to accept or reject this resource request. In Configuration model (e.g. COPS-PR for Diffserv), the PEP installs policies before the arrival of resource request event. When a resource request arrives (e.g. a Diffserv packet arrives at a Diffserv router), the PEP enforces installed policies (e.g. remark the DiffServ Code Point, put a packet in besteffort queue...) without sending a REQ message to the PDP.

COPS protocol itself defines 10 messages and objects only for general purpose of policy information transport. In each policy domain, where COPS is used for a specific purpose, an extension must be defined and distinguished by the Client-Type field in the common header of COPS message. There are many extensions of COPS protocol but only COPSRSVP [4] is assigned by IANA with a 'Client-Type $=1$ '. Other extensions, such as COPS-PR for DiffServ router configuration [5], COPS usage for IP Traffic Engineering [6], COPS usage for IPsec configuration [7], are currently works in progress.

COPS-SLS Service Level Specification (SLS) [15] is a set of parameters and their values which together define a service offered to a traffic stream. It is the technical part of the Service Level Agreement (SLA). To negotiate a 
level of service with an ISP (Internet Service Provider), the client sends its desired SLS to the network. The network can accept or reject the request, or propose another level of service to the client.

COPS-SLS [2] is an extension of COPS protocol. The idea of COPS-SLS is to apply Policy-based networking in SLS negotiation. This extension gives I3 platform a means to negotiate dynamically between a client and a network provider about client's needs, such as QoS parameters, security, mobility, etc. Figure 2 presents the COPS-SLS model.

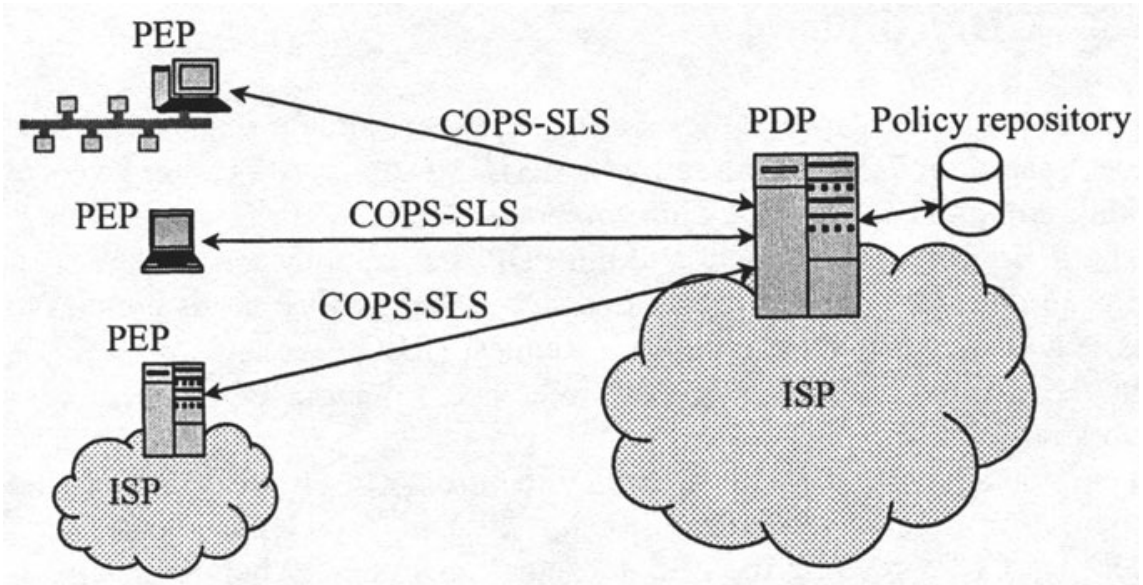

Figure 2. COPS-SLS Model

The SLS-PDP entity represents the network provider and the SLS-PEP entity represents the client. The client here is just a logical entity who requests, possibly on behalf of other entities, network resources. It may be an end-host (connected to the network via a modem), or a gateway of a local network, or another ISP. The fact that the PEP may be put in an end-host broadens the working domain of Policy-based Networking while earlier systems consider the PEP only as network element. We have not only policies to manage network devices in a domain, but also policies to manage each type of client in the domain.

COPS-SLS comprises two phases: Configuration phase and Negotiation phase. The organization of SLS negotiation process in two phases makes the negotiation very dynamic. The Configuration phase decides how the Negotiation phase takes place. For example, the network supplies the client with information about the negotiation mode, time interval to renegotiate, PIB classes to be exchanged, etc. [2]. After successfully installing the configuration supplied by the PDP, the PEP can start the Negotiation phase 
by sending a request for its desired level of service. The PDP can accept or reject the request or propose another level of service to the client. The PEP installs policies in the decision and sends a report. If both decision and report are positives, the contract is established and the user data obtain the negotiated level of service. At any time, the network can send an unsolicited decision to change the parameters of the Negotiation phase. This phase will be guided with the updated configuration.

SLS information exchanged between the PEP and the PDP is represented by a named data structure, a.k.a. a PIB [3]. The use of a PIB for a SLS representation gives a possibility to take into account all desired negotiation parameters of all providers. Common classes may be used to specify common parameters of the service and private classes may be used by network providers to personalize their SLS parameters.

For intra-domain application, COPS-SLS can be used to manage user levels of service in the domain. For inter-domain application, it is interesting to establish and manage level of service of inter-domain communications. We hope that COPS-SLS can make the SLA really dynamic both between the client and the network and between ISPs.

\section{MOBILITY AND COPS-MU}

One aspect the platform I3 has to deal with is user mobility management. In this section, we present an extension of COPS protocol to deal with such a issue.

User mobility [8] concerns the terminal mobility and the personal mobility, which is the ability of the user to use different terminals and access his personal services in his home network from anywhere [9].

We identify four issues in the user mobility management which are: terminal registration, user registration, services portability and QoS negotiation We propose to have a policy enforcement point (PEP) on the terminal called Terminal PEP (TPEP) which interacts directly with the Foreign PDP (FPDP) for terminal registration, user registration, services portability and QoS negotiation.

\subsection{Terminal and user registration}

Terminal registration must be achieved only if a terminal is located in a foreign network, if it is a fixed terminal or a mobile terminal located in its home network then terminal registration is not needed. A user registration must be achieved every time a user logs in a terminal even if a user is in his home network 
Terminal registration consists of maintaining an association in the Terminal Home Agent (THA) between the terminal Care of Address (CoA) and its home address. User registration consists of maintaining an association in the User Home Agent (UHA) between the user identifier and the IP address of a terminal he is using. The user mobility binding allows to the user to be reachable on the terminal he is using, and to use subscribed personal home network services from anywhere. The later deals with the service portability management.

Steps explained below are the suggested steps for policy based terminal and user registration management illustrated in Figure 3:

1. TPEP interacts directly with the Terminal Foreign PDP or TFPDP (rep. User Foreign PDP or UFPDP) for terminal (albeit user) registration request policy decisions;

2. Mobile Terminal (MT) or the TPEP sends a registration request to the THA (albeit UHA);

3. Terminal Home PEP or THPEP (albeit User Home PEP or UHPEP) interacts with the Terminal Home PDP or THPDP (albeit User Home PDP or UHPDP) for terminal registration request (albeit User registration request);

4. HA sends registration reply message to the MT;

5. TPEP interacts with TFPDP (albeit UFPDP) for terminal (albeit user) registration reply policy decisions.

In this scenario the terminal has a co-located CoA such as in IPv6 wich is a routable adress. If the terminal has a CoA, it achieves it registration through the FA [10].

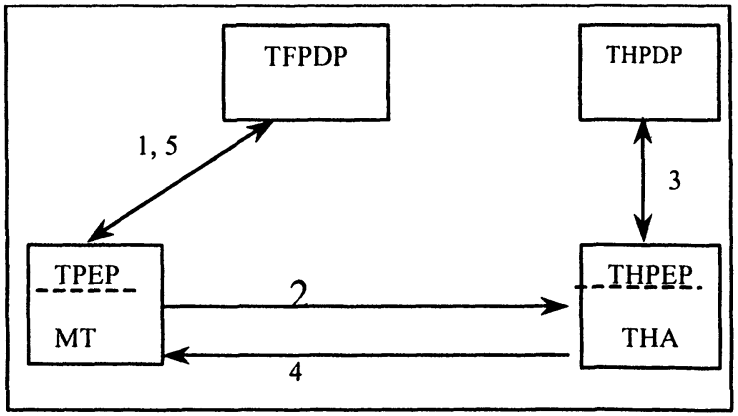

Figure 3. Policy-based Terminal registration 


\subsection{Service portability}

Service portability is related to using personal home network services from anywhere and from any terminal. Third generation of mobile networks deals with service portability using Virtual Home Environment (VHE).

We suggest using a policy concept to deal with service portability in IP networks. Thus, TPEP has to interact with FPDP, UHPDP, and THPDP for service portability and QoS negotiation. This negotiation may be achieved either at a same time with the terminal and user registration or, at any time when a user needs to use a home or a foreign network service.

In a wireless network access we have to minimize traffic on a wireless link. We suggest reporting negotiation process in a fixed part of a network. TPEP interacts only with one PDP; either a HPDP or a FPDP, and make PDPs negotiating to support the user requests for service portability.

Figure 4 illustrates personal home network service portability negotiation. Numbered steps on this figure are explained bellow:

1. TPEP interacts with a UHPDP for service portability. TPEP sends to a UHPDP:

- the terminal profile;

- the required home service.

2. UHPDP interacts with its UHA to determine the required user service features using user profile services. UHPDP based on previous criteria interacts with FPDP and THPDP to decide where to carry out a required home service. FPDP sends to UHPDP its:

- network access features which are related to the QoS that may be offered by a fixed or wireless network;

- foreign network access resources and services cost.

THPDP sends to UHPDP the terminal profile if it is not sent by the TPEP.

3. Based on UHPDP policy decisions, UHPDP may interact with UHPEP to configure its local resources using UHPEP to provide user home services in the home network, FPDP may interact with FPEP to configure its local resources to support personal home service portability, and THPDP may interact with TPEP to configure the MT to support personal service portability. 


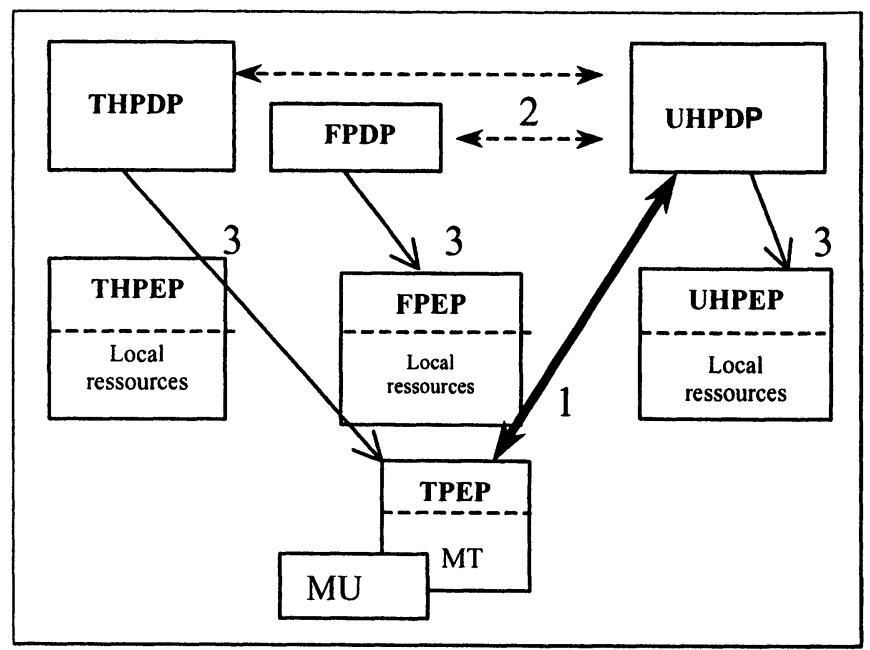

Figure 4. Policy-Based service portability management

QoS parameters defined in fixed networks are: bandwidth, delay, jitter, and, packet loss. User mobility also defines other parameters related to mobility management such as packet loss due to handoff, and, handoff blockage probability. When a MU needs to use one service with a required QoS, the TPEP interacts with the FPDP for QoS negotiation. We are working on the extension of the wired access networks QoS negotiation protocol COPS-SLS presented in the previous section to support the negotiation of QoS in wireless access networks.

\subsection{COPS-MU policy objects}

In the policy based user mobility management we need to define these objects:

- Objects defined in Mobile IP related to terminal registration;

- New Objects defined in extended Mobile IP to support user registration;

- New Objects defined to transport terminal parameters, access network parameters, personal services parameters, user mobility behaviour for service portability and QoS negotiation.

It is important to note that COPS support security mechanisms such as authentication and data integrity. These features are interesting in the user mobility management. Paper [11] suggests that the authentication procedure for Mobile IP can be achieved by another entity than HA or FA. In our model, we suggest that the authentication procedure will be supported by the policy server PDP. Thus, we have to define authentication objects to achieve Terminal PEP, Foreign PEP, and Foreign PDP authentication, and Terminal 
PEP, Foreign PEP and Terminal Home PDP authentication, and Terminal PEP, Foreign PEP, and User Home PDP authentication.

Presently, we are defining a new PIB (Policy Information Base) [12] to support mobile user policy objects structure.

\section{I3 MODEL}

The aim of $\mathrm{I} 3$ project is to put within the core network some intelligence to get easier its management. This intelligence will be introduced through agents in network equipments.

In $\mathrm{I} 3$ project, a policy-based architecture is considered. In addition, each flow associated to a client is characterised by a profile. The definition of a profile is quite similar to SLA notion, but goes beyond the classical vision characterising a traffic flow. Indeed, a profile, characterising the client's needs, contains a set of requirements: QoS parameters, security needs, mobility requirements, etc.

\subsection{A Functional Architecture supporting QoS}

In order to meet the main requirements (QoS) of 13 platform, we propose the functional architecture shown in Figure 7. There are four main parts in this architecture: Contracts Management, Network Management, Policy Management and Monitoring. 


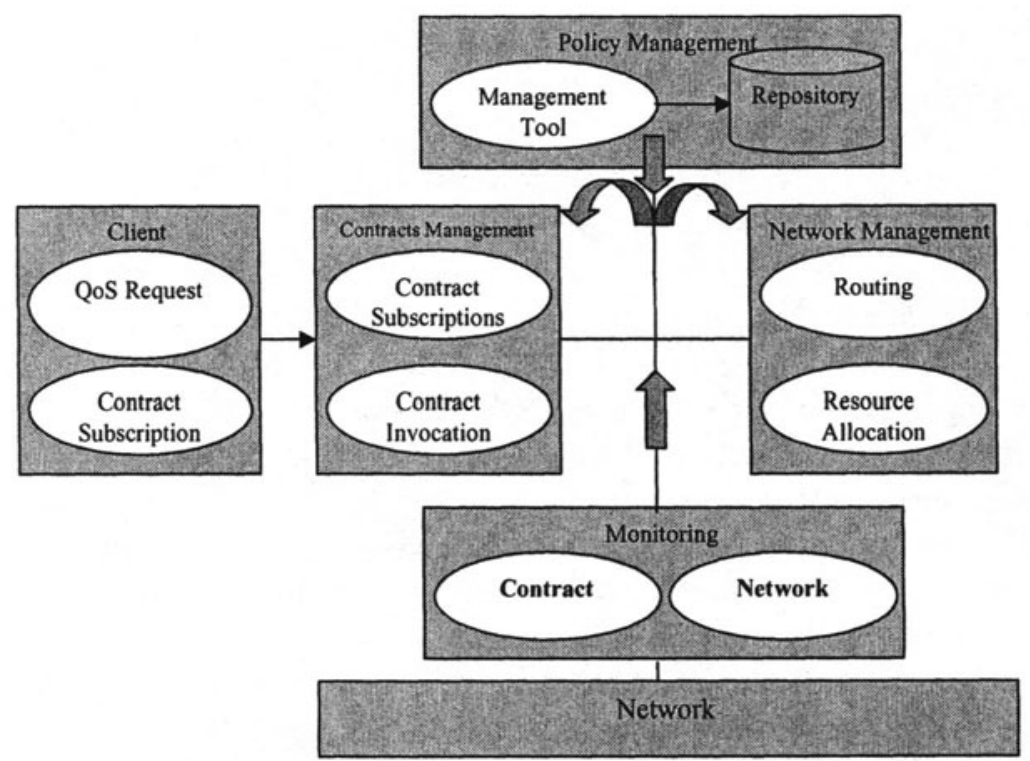

Figure 5. The functional architecture

Contracts Management is responsible for all contracts-related activities and includes two functions: Contract Subscription and Contract Invocation. Contract Subscription is responsible for customer registration and policybased admission. The customer might either be an autonomous system, a PDP of another domain or a residential customer. Contract Invocation is in charge of admission control as requested by the user.

Network Management is responsible for mapping the traffic onto the physical network resources and configures the network according to the contract. Two sub-functions may be determined: routing and resource reservation.

Policy Management includes functions such as Policy Management Tool and policy Storing Service.

Finally, Monitoring provides information about the resource usage and the quality of offered services. Monitoring has not only a diagnostic role but also it has a pro-active/reactive operational role $[13,14]$.

\section{$5.2 \quad$ I3 platform}

The goal of $\mathrm{I} 3$ is to put the intelligence within the network by means of agents in order to ensure the functionalities described above. 
The organization of activities of the defined agents is necessary. Madkit platform is the candidate platform we have chosen to implement 13 environment. Indeed, Madkit is based on AALAADIN model which provides simple and interesting abstractions allowing agent's activities and agent's interaction modeling.

\subsubsection{AALAADIN MODEL AND MADKIT PLATFORM}

AALAADIN is the organizational description model that structures MADKit platform [16]. This model is based on three main abstractions (Figure 5)

\section{Agent}

There are no constraints on the internal architecture of an agent. An agent is just specified as an active communicating entity, playing roles within groups. This definition is left general to allow agent designers to adopt the most accurate definition of "agenthood" according to their application. The agent designer is responsible for defining the most appropriate agent model he needs.

\section{Group}

Groups are defined as the smallest agent aggregation. An agent is part of one or several groups. The group is only a way to tag a set of agents. In a more developed form, in conjunction with the role definition, the group may represent any usual multi-agent system. An agent can be a member of several groups at the same time. Moreover, AALAADIN groups can freely overlap. Any agent can found a group, and an agent must request its admission to an existing group. Groups might be distributed among several machines.

\section{Role}

The role is an abstract representation of an agent function, service or identification within a group. Each agent can own several roles, and each role handled by an agent is local to a group. As with group admission, handling a role in a group must be requested by the candidate agent, and is not necessarily accepted. 


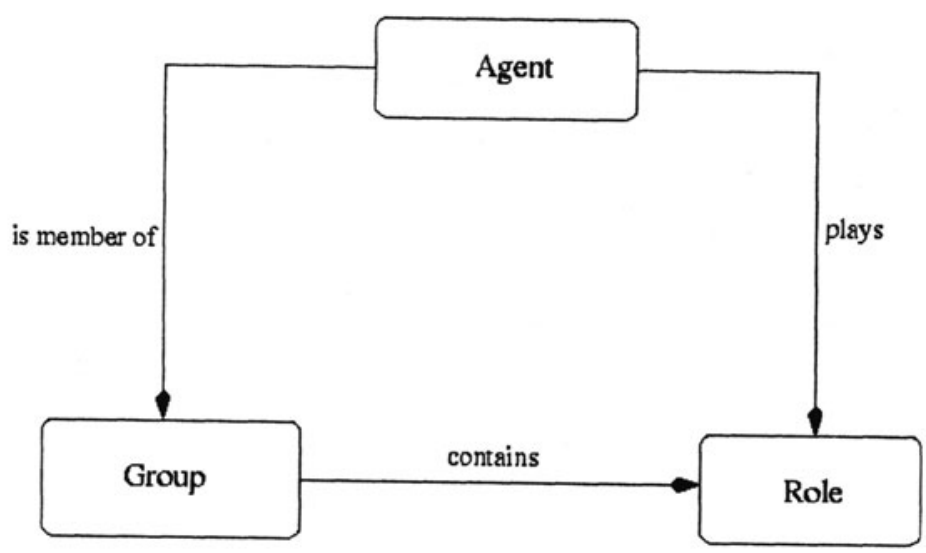

Figure 6. AALAADIN Model basic Abstraction

To communicate, two agents should own a role within the same group. But it is possible to communicate through agents, as shown in Figure 6. In this example, agents A and D cannot directly communicate. Agents A and B own the same group 1 and then are able to communicate directly. Agents B and $\mathrm{C}$ own the group 2, and agents $\mathrm{C}$ and $\mathrm{D}$ the group 3 . Then, it is possible to make communicating agents $\mathrm{A}$ and $\mathrm{D}$ through agents $\mathrm{B}$ and $\mathrm{C}$, according to the established hierarchy.

It is worth stressing that an agent can be a member of at least one group, a group contains at least one role, and an agent plays at least one role within one group.

MADKit stands for "Multi Agent Development Kit". It is an agent platform developed at LIRMM laboratory. Written in Java, this platform is easy to use on several system environments.

MADKit is based on AALAADIN organizational model to manage its agents. MADKit platform has been chosen to implement the prototype of $\mathrm{I} 3$ project for two main reasons. MADKit agents allow adding some intelligence within network elements and the organizational model makes easier the handling and management of network streams. An agent is a small program written in Java. MADKit platform is used through an API (Application Programming Interface) providing classes and methods allowing an organizational management of the agents. Abstractions as "group" and "role" help during the creation of the agents, giving them a logical role. Classes of MADKit API make easier the communication between agents. For example, it is possible to broadcast a message to all agents of a given group, or to all agents owning the same role within a given group. Other Java methods are defined to ensure the management of roles (add, remove, etc.). 


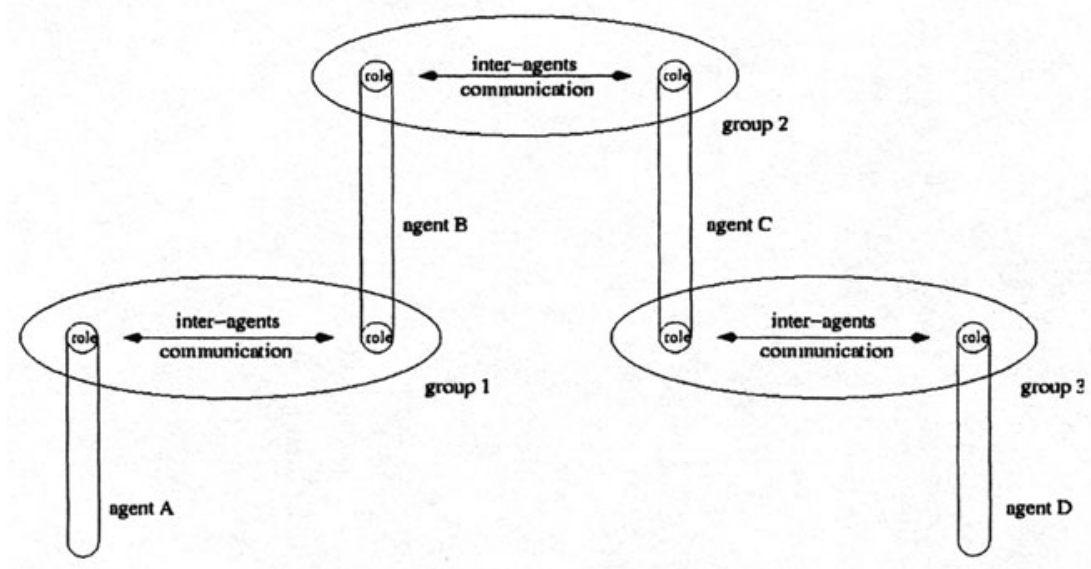

Figure 7. Inter-Agent Communication

\subsubsection{I3 PLATFORM MODEL}

\section{a. The PEP Model}

Figure 8 represents the AALADIN model of the I3 platform. Each "I3 router" is considered as a PEP. The PDP handles the management of network resources and the enforcement of decisions within the PEP so that the client (flow) requirements are satisfied. The I 3 router model includes the following components:

\section{The StreamAgent}

The StreamAgent, associated with each flow stream, is defined to handle the flow during the communication live time. The main goal of StreamAgent is to provide the platform with information about flow requirements $(\mathrm{QoS}$, security, mobility). For each requirement a group is defined. Thus, if a flow needs a given requirement the corresponding StreamAgent owns a given role within the corresponding group. For example, the StreamAgent could have a "maximum_security" role, in a security group, and a "low_mobility" role, in a mobility group. These roles characterize the stream, making easier its management. The set of flows having the same requirements are aggregated and managed by the same agent i.e., the ManagerAgent. 


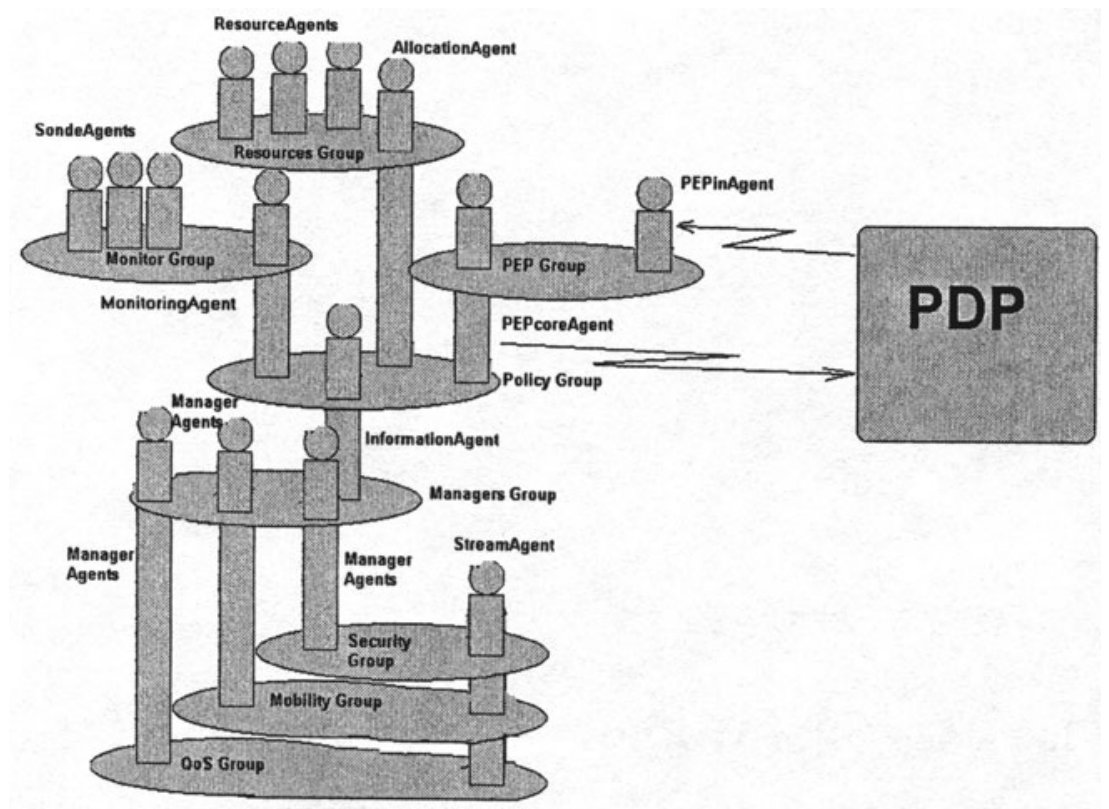

Figure 8. I3 Platform model

\section{The InformationAgent}

The InfomationAgent has a global vision about all client's needs. It submits the service request to the PDP server via a "Policy" and "PEP" groups. The PDP Server decides on the policies to be enforced to satisfy the needs. In particular, the decisions may concern resource allocation. In this case, the request is taken in charge by the AllocationAgent and forwarded to the ResourceAgents, which represent the network resources.

\section{The MonitoringAgent}

The MonitoringAgent is the manager of the "monitor" group, which contains Agents for collecting indicators of QoS.

\section{b. The PDP model}

It is worth stressing that the PDP server is a remote entity. Thus, the communication between a PEP and a PDP does not take place in the same group. Intermediate agents, i.e., PEPCoreAgent and PEPInAgent, which communicate via COPS messages, are defined (see Figure 9). 


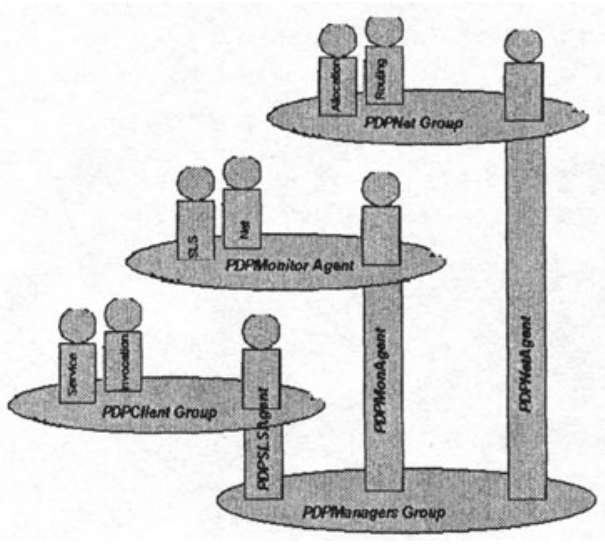

Figure 9. PDP model

\section{c. The client model}

In addition to the model defined for the PDP/PEP environment, we propose a client model (see Figure 10) for the choice of the provider. Indeed, the main problem encountered by the operators and providers is at the level of their access network that generally constitutes the bottleneck. We retained the protocol called "Contract Net" based on the concept of contracts between Providers (ISP) and consumers (Customer) of services. We have introduced a broker which is connected to all the providers and who plays the role of an edge router. Thus each customer knows only the address of the broker.

In this model, provider agents take suitable roles in the Provider-Group according to their competence, and attribute prices for their Services. An Agent Broker joints the Client-Group group and the Provider-Group. When a customer wants to make a request for service with a given QoS, a Client agent joints the Client-Group group with the role of member. It seeks for the Broker role, and asks him to find the best offers for the requested QoS (for example, a Gold, Silver or Bronze Assured Forwarding service in DiffServ). The broker agent interacts with the provider concerned with the ProviderGroup, chooses the best offers and transmits information to the Client and to the selected Provider. Then, these two agents create their own ContractGroup group to sign the contract. 


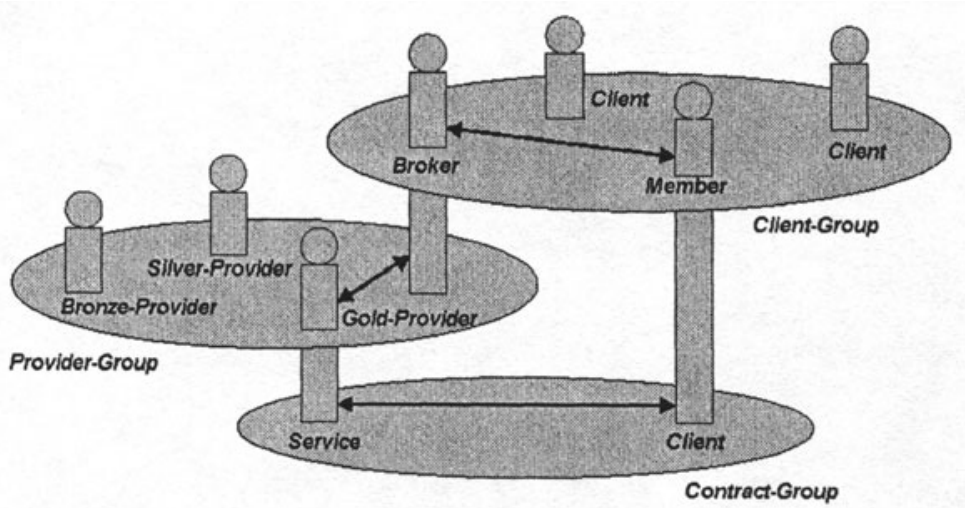

Figure 10. Agent model

\section{CONCLUSIONS}

This paper introduced new concepts to support future Internet generations. These concepts are based on the introduction of some intelligence in a policy-based networking environment.

We believe that the use of COPS and PDP/ PEP model offers a good way to achieve IP network management. In the case of user mobility, it could allow the deployment of universal personal computing policy control in IP networks by offering policy based network providers negotiation for user and terminal management, service portability, and QoS negotiation.

We proposed a policy-based networking environment supporting new protocols compatible with COPS and simplifying the control of the QoS and the mobility in the IP network. Then, we describe the I3 platform able to support some intelligence to help the new policy-based networking environment to react, manage and control future Internet networks.

Future works concern authentication and more generally the security to be provided in the future IP networks.

\section{REFERENCES}

[1] D. Durham, Ed., J. Boyle, R. Cohen, S. Herzog, R. Rajan, A. Sastry, "The COPS (Common Open Policy Service) Protocol", RFC 2748, January 2000.

[2] T.M.T. Nguyen, G. Pujolle, N. Boukhatem, "COPS Usage for SLS negotiation (COPS-SLS)", draft-nguyen-rap-cops-sls-00.txt, work in progress, June 2001. 
[3] K. Chan, J. Seligson, D. Durham, S. Gai, K. McCloghrie, S. Herzog, F. Reichmeyer, R. Yavatkar, A. Smith, "COPS Usage for Policy Provisioning (COPS-PR)", RFC 3084, March 2001.

[4] S. Herzog, Ed., J. Boyle, R. Cohen, D. Durham, R. Rajan, A. Sastry, "COPS usage for RSVP", RFC 2749, January 2000.

[5] M. Fine, K. McCloghrie, J. Seligson, K. Chan, S. Hahn, C. Bell, A. Smith, Francis Reichmeyer, "Differentiated Services Quality of Service Policy Information Base", draft-ietf-diffserv-pib-03.txt, work in progress, March 2001.

[6] C. Jacquenet, "A COPS client-type for IP traffic engineering", draft-jacquenetip-te-cops-02.txt, work in progress, June 2001.

[7] Man Li, David Arneson, Avri Doria, Jamie Jason, Cliff Wang, "IPSec Policy Information Base", draft-ietf-ipsp-ipsecpib-03.txt, work in progress, July 2001.

[8] E. Koukoutsis, C. Kossidas, N. Polydorou, "User Aspects for Mobility", Acts Guideline SII-G8/0798.

[9] A. Fasbender,F. Reichert, E. Gueulen, J. Hjelm, T. Wierelemann, "Any Network, Any Terminal, Anywhere", IEEE Personal Communications 1999.

[10]C. Perkins, "IP mobility support", RFC 2002, October 1996.

[11]L. Bos, S. Leroy, "Toward an All-IP-Based UMTS System Architecture", IEEE Network, January 2001.

[12]B. Moore, et al, "Policy Core Information Model", RFC 3060, February 2001.

[13] Tequila, "A Management and Control Architecture for Providing IP Differentiated Services in MPLS-Based Networks", IEEE Communications, May 2001.

[14]Tequila, "A Monitoring and Measurement Architecture for Traffic Engineered IP Networks", IEEE Communications, May 2001.

[15]D. Goderis, Y. T'joens, C. Jacquenet, G. Memenios, G. Pavlou, R. Egan, D. Griffin, P. Georgatsos, L. Georgiadis, P. V. Heuven, "Service Level Specification Semantics, Parameters and negotiation requirements", drafttequila-sls-01.txt, work in progress, June 2001

[16] O. Gutknecht, J. Ferber, "Madkit : Organizing heterogeneity with groups in a platform for multiple multi-agents systems", Technical Report 97188 LIRMM, Montpellier, 1997 http://www.lirmm.fr/ gutkneco/aalaadin/rr97188.ps.gz) 